\title{
Genetics in Cleft Lip and Palate
}

\author{
Dr. Sathya Ramanathan, Dr. Umashankar
}

\begin{abstract}
Orofacial cleft is one of the most significant birth defects across the globe. The focus of this work is to highlight the most recent advances in understanding of cleft lip and palate occurrence. Information regarding research on long time outcomes, genes, and their interactions with other genes are compiled to provide the overview on the current knowledge of the etiology of cleft lip and palate .A gene IRF6(interferon regulatory factor) has been shown to be an important contributor to cleft lip and palate ,but the functional variant leading to the defect has not yet been defined. Inactivation of the gene MSXI and genes in the FGF(fibroblast growth factor) family have also been shown to cause cleft lip and palate in human beings. In addition, mutations in several candidate genes may cause cleft lip and palate, but definitive evidence regarding the biological consequences of the mutations is yet to be unravelled. The recent advances in cleft research includes the analysis of several additional phenotypical features of the population, with the goal of increasing the statistical power of genetic studies.
\end{abstract}

Keywords: cleft lip palate, IRF6, genes, mutation, advance

\section{Introduction}

Orofacial clefting is a marked anomaly which manifests in a new born individual. Orofacial cleft involves structures around the oral cavity which can extend on to the facial structures resulting in oral facial and craniofacial deformity.

\subsection{Rationale}

India is one of the many regions of the world, where cleft lip and palate occur in a very frequent manner. Documentation of the rates of birth anomalies is incomplete due to the density of the population. Many parents in India of a child born with cleft lip and palate have no access to counseling on care and treatment of their affected children. The lack of knowledge and resource results in unacceptable delays in seeking and receiving adequate medical care due to which many infants with Orofacialcleft are left with permanent diability.

\subsection{Experimental Design}

Clefts involving the lip and palate are a significant congenital anomaly. This requires a complex long term treatment and has a complex phenotype which reflects a break-down in normal mechanism involved during embryological development of the face. The incidence of these defects varies according to geographical locations, ethnicity and socio economic status.

Broadly speaking, approximately $70 \%$ of Cleft lip and palate, cases are non syndromic and remaining $30 \%$ are syndromic cases with structural abnormalities.

Our understanding of the etiology and pathogenesis still remains relatively poor. This is a reflection of the complexity and diversity of the mechanisms involved at molecular level during embryogenesis with both genetic and environmental factors playing an important and influential role.

With recent advances in genetics, progress has been made in identifying genes associated with this anomaly and their influence in the development of the facial complex.

\subsection{Objectives}

Many families display a definite hereditary tendency of cleft lip and palate individuals, but do not show specific mode of inheritance. One reason is probably an indefinite designation and definition of phenotype for clefting genes. Such varying phenotypes can be due to :-

\section{a ) Minor anatomical discrepancies.}

\section{b) Genetic heterozygosity}

The purpose of this study is to identify and to determine the prevalence of orofacial variations which are possible incomplete manifestations of gene action. This would permit better definition of the phenotype of cleft lip and palate and thereby help to clarify any role these microforms might play in delineating a specific mode of inheritance. 


\subsection{Review Of Litrature}

- A systematic review reported a data which consisted of 51 families with cleft lip and palate .DNA samples were extracted. There were evidence for altered transmission of IRF6 gene.(1)

- A case control study of 763 facial clefts(CL/P) in which they genotyped 6 nucleotide polymorphisims within the IRF6 locus and estimated the relative risks(3)

- A case study of 83 cleft lip palate cases where they had clinical features of Van der Woude syndrome which encompasses the interferon regulatory factor 6(IRF6) and FGFR2 gene(2)

- Case study report of $184 \mathrm{CL} / \mathrm{P}$ patients says point mutations in genes FOXE1,MSX2 appear in bilateral cleft lip and palate and positive family history.(4)

- Systematic review on 45 Thai patients with cleft lip and palate stated that it is caused by mutations in TBX22 TRANSCRIPTION FACTOR(5)

- Another case study says DNA samples isolated from individuals born with cleft lip palate showed that array-CGH analysis of DNA samples is an efficient and productive method for identifying candidate chromosomal loci and genes-ESR1 and FGFR2(6)

\subsection{Methodology}

1 Study of Population

2 Identifying Genes using Gene mapping strategies.

3 Stem Cell cultures.

\section{Population Studies Of Cleft Lip And Palate}

- Epidemiological approach.

According to US bureau of census

$2001-1$ in 600 new borns were affected with cleft anomaly.

Assuming 15000 births per hour worldwide a child is born with a cleft somewhere in the world every 2 minutes From 1960 to 1979 :

Out of 4,07,025 births the occurrence of Cleft lip and Palate was diagnosed in 440 cases that is 1.08 affected individuals per thousand births.

Out of the 440 cases diagnosed, 95 individuals were affected with Cleft Palate that is.0.25 of the affected individuals per thousand births.

\subsection{Prospective Study Of Malformations In 17 Centres All Over India ( Sep 1989 To 1990).}

Out of 47,787 births the occurrence of Cleft lip and Palate was diagnosed in 64 cases that is 1.3 affected individuals per 1000 births.

Out of the 64 cases diagnosed, 6 cases were affected with Cleft Palate that is , 0.12 affected individuals per 1000 births.

\section{During The Period Of 1994-1996:-}

Out of 94,610 births in the 3 cities namely Baroda, Delhi and Mumbai . The occurrence of Cleft lip was 0.93 individuals per 1000 and Cleft Palate was 0.17 individuals per 1000 births.

This means that 78 of the total born everyday are affected infants and among that 3 infants are born with Cleft every hour.

We deduce from these epidemiological studies that these counseling centres will be able to evaluate their own quality of care and compare with other centres to enable implementation of the local quality programe and improvement.

The first step towards attainment of minimum standards of care has been encouraged on type and timing of record collection of statistical data for the measurement of outcome. This will result in accumulation of wealth of data that can be used for inter centre comparisons both for research and for audit in future.

\section{Gene Mapping Strategies:-}

Gene mapping was carried out using Blood or tissue samples from family members where cleft lip and palate are prevalent .

DNA was isolated from these samples and examined for unique pattern of bases seen in family members. The characteristic molecular patterns are referred to as Markers.These DNA markers can tell roughly where the gene is located on the chromosome. This is possible by recombination techniquesThe gene close to DNA markers will adhere together during recombination process and be passed on together from the parent to the child. 


\section{Str Selection And Pcr Amplification:-}

Genomic DNA was extracted from $200 \mu 1$ of peripheral blood with the use of genomic DNA isolation kit. Multiplex PCR amplification was performed with microsatellite amplification kit with a total volume of 25 $\mu 1$ of blood. The amplifications were subjected to electrophoresis on an ABI prism 3100 genetic analyser and analysed by genescan version 3.0 and genotype version 2.1 software (applied biosystems).

\subsection{Implications}

The need on importance of birth defect surveillance and research is required for:-

1 Establishment of birth prevalence of craniofacial anomalies.

2 Improving the quality of care.

3 Determining the genetic and environmental etiology of cleft.

Identifying the gene linked in a genetic disease, opens a way to several lines of investigation. The ability to identify mutation, should immediately lead to improved diagnosis and counseling.

Understanding the molecular pathology may also lead to insight into related diseases eventually leading to more effective treatment including gene therapy.

Isolation of genes in childhood itself can prevent its transmission to the future generations.

\section{Conclusion}

Cleft lip and palate is the most common facial birth defect and it is caused by a complex interaction between genetic and environmental factors. The purpose of this review is to provide an overview of the spectrum of the genetic causes for cleft lip and palate. Although the gene identification process for orofacial clefting In humans is in the early stages, the pace is rapidly accelerating. Recently several genes have been identified. Ongoing human genome -wide linkage studies have identified regions in genome that likely contain genes that when mutated cause orofacial clefting,including a major gene on chromosome 9 that is positive in multiple racial groups. Furthermore, statistical geneticists are developing new methods to characterize both gene-gene and gene-environment interactions to build better models for pathogenesis of this common birth defect. The ultimate goal of these studies is to provide knowledge for more accurate risk counseling and development of preventive therapies.

\section{Reference}

[1]. Susan H. Blanton, Amy Cortez, Jacqueline T.Hecht Variation in IRF6 contributes to nonsyndromic cleft lip and palate. 2005 DOI: 10.1002/ajmg.a.30887

[2]. K Osoegawa ,G.M Vessere, A.Borg ,J.C Murray Identification of novel candidate genes associated with cleft lip and palate using array comparative genomic hybridization. J Med Genet 2008:45:81-86 doi:10.1136/jmg.2007.055219

[3]. Astanand Jugessur ,Fedik Rahimov, Jeffrey C.Murray Genetic variants in IRF6 and risk of facial clefts: single marker and haplotype-based analyses in a population-based case control study of facial clefts in Norway. 2008 DOI: 10.1002/gepi.20314

[4]. Alexandre R Viera, Joseph R Avila, Jill Harrington Medical Sequencing of candidate genes for Nonsyndromic cleft lip and palate. 2005.DOI:10.1371/JOURNAL.PGEN.0010064

[5]. Brewer C ,Holloways S , Chromosomal deletion map of human malformations. Am J Hum Genet.1998:63:1153-1159

[6]. Cardon LR , Palmer LJ ,Population stratification and spurious allelic association.Lancet.20033:361:598-604

[7]. Cynthia H. Cassell, Julie Daniels and Robert E. Meyer. (2009) Timeliness of Primary Cleft Lip/Palate Surgery. The Cleft PalateCraniofacial Journal 46:6, 588-597.

[8]. Traci Flynn, Christina Persson, Claes Moller, Anette Lohmander and Lennart Magnusson. (2014) A Longitudinal Study of Hearing and Middle Ear Status of Individuals With Cleft Palate With and Without Additional Malformations/Syndromes. The Cleft PalateCraniofacial Journal 51:5, e94-e101

[9]. Agneta Marcusson, Ingemar Akerlind and Gunnar Paulin. (2009) Quality of Life in Adults With Repaired Complete Cleft Lip and Palate. The Cleft Palate-Craniofacial Journal 38:4, 379-385.

[10]. Victoria S. Lucas, Ragini Gupta, Olubenga Ololade, Marilyn Gelbier and Graham J. Roberts. (2009) Dental Health Indices and Caries Associated Microflora in Children With Unilateral Cleft Lip and Palate. The Cleft Palate-Craniofacial Journal 37:5, 447-452. Online publication date: 1 -Sep-2000 